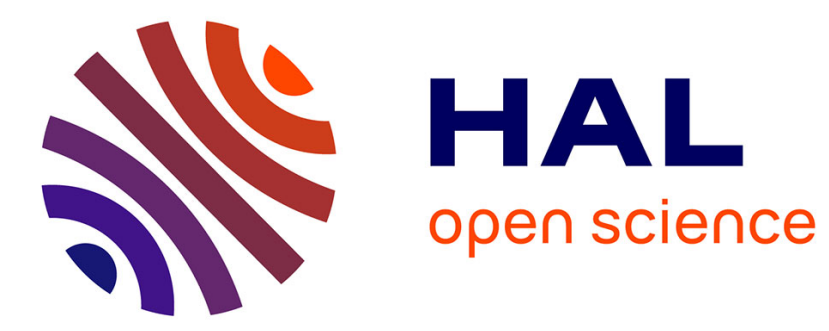

\title{
Frustration in periodic systems : exact results for some 2D Ising models
}

\author{
G. André, R. Bidaux, J.-P. Carton, R. Conte, L. de Seze
}

\section{To cite this version:}

G. André, R. Bidaux, J.-P. Carton, R. Conte, L. de Seze. Frustration in periodic systems: exact results for some 2D Ising models. Journal de Physique, 1979, 40 (5), pp.479-488. 10.1051/jphys:01979004005047900 . jpa-00209129

\section{HAL Id: jpa-00209129 https://hal.science/jpa-00209129}

Submitted on 1 Jan 1979

HAL is a multi-disciplinary open access archive for the deposit and dissemination of scientific research documents, whether they are published or not. The documents may come from teaching and research institutions in France or abroad, or from public or private research centers.
L'archive ouverte pluridisciplinaire HAL, est destinée au dépôt et à la diffusion de documents scientifiques de niveau recherche, publiés ou non, émanant des établissements d'enseignement et de recherche français ou étrangers, des laboratoires publics ou privés. 


\title{
Frustration in periodic systems : exact results for some 2D Ising models
}

\author{
G. André, R. Bidaux, J.-P. Carton, R. Conte and L. de Seze \\ DPh-G/PSRM, CEN Saclay, B.P. 2, 91190 Gif-sur-Yvette, France \\ (Reçu le 30 novembre 1978, accepté le 12 janvier 1979)
}

\begin{abstract}
Résumé. - On présente un certain nombre de résultats exacts concernant des modèles d'Ising frustrés à deux dimensions avec interactions périodiques. Aucun d'entre eux ne donne lieu à ce qui pourrait être considéré comme un ordre verre de spin. Les seuls ordres à longue distance rencontrés sont de type ferro- ou antiferromagnétique ; toutefois l'effet de la frustration se manifeste à basse température (entropie, susceptibilités). L'analyse de ces résultats fournit quelques indications sur une condition nécessaire pour que de tels systèmes s'ordonnent, ainsi que la nature possible de l'ordre verre de spin.
\end{abstract}

Abstract. - We present exact results for various frustrated Ising models in two dimensions with periodic interactions. None of them gives rise to what could be regarded as spin glass order. The only long range orders encountered are of ferro- or antiferromagnetic type but the effect of frustration manifests itself at low temperature (entropy, susceptibilities). The analysis of these results provides some indications concerning a necessary condition for ordering in such systems and the possible nature of the spin glass order.

Introduction. - The recent interest in the theory of spin glasses has drawn attention to so-called frustration effects, which are regarded as the relevant concept in these problems (at least for Ising systems) as pointed out by Toulouse [1]. In particular the question arises whether some spin glass state may exist in regular, periodic, frustrated lattices, since the spin glass properties are expected to be due to frustration only.

Owing to the uncertainty that prevails in this field, exactly soluble models are of the highest interest. Unfortunately the only models of this type solved so far (to our knowledge) are Villain's odd model [2] (i.e. the 2-dimensional totally frustrated Ising model) and the antiferromagnetic triangular Ising model solved by Wannier [4] : neither of them shows a phase transition $\left({ }^{1}\right)$.

This led us to consider two generalized versions of the odd model, allowing different strengths of interaction. Phase diagrams are obtained for both. We also consider some cases of periodically distributed frustrated plaquettes. Our results suggest that regular models may lead to ferromagnetic ordering, or antiferromagnetic ordering or no ordering at all but never show spin glass order in two dimensions.

( ${ }^{1}$ ) Actually some of the models studied by Villain do have a transition in the $\mathrm{XY}$ version for which however the concept of frustration has very different implications from the Ising case.
1. Definition of the dominoes models. - We consider Ising models on a square lattice with nearest neighbours interactions as shown in figure 1. Because the elementary cell looks like a domino, the first model will be called piled up dominoes model (PUD), and the second zig-zag dominoes model (ZZD). Throughout the paper $J$ will be taken positive.

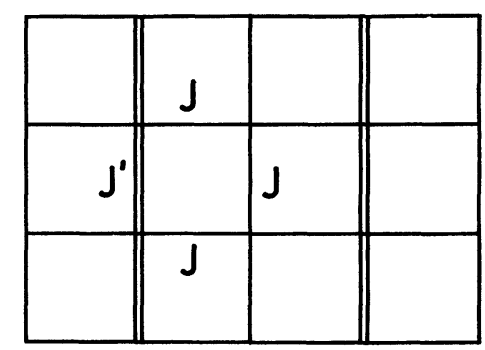

a)

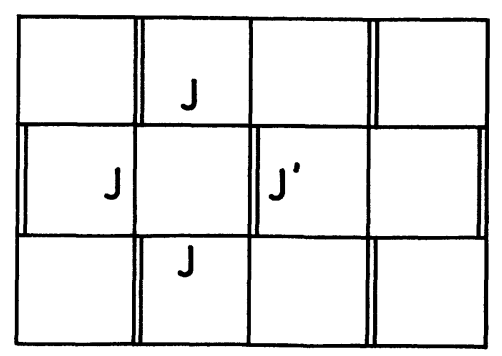

b)

Fig. $1 .-a$ ) Interactions in the PUD model; $b$ ) Interactions in the ZZD model. 
When $J^{\prime}=-J$ both models are equivalent through a gauge or Mattis transform : this is the odd model introduced by Villain.

In the case of the PUD model, for clarity, the vertical strings along which the interactions are $J$ (resp. $J^{\prime}$ ) will be called even strings (resp. odd strings).

2. Solution of the piled up dominoes model. 2.1 LOW-TEMPERATURE PROPERTIES. - i) $J^{\prime}>-J$ : it is clear that the ground state is the ferromagnetic state.

ii) $J^{\prime}=-J$ : Villain [2] has shown that the ground state is degenerate and yields a finite entropy, equal to the entropy of the dimer model on the dual lattice. We will later return to this case ( 33.2 and $\S 4)$.

iii) $J^{\prime}<-J$ : in the ground state the $J^{\prime}$ bonds are necessarily satisfied and the $J$ bonds only may be frustrated. We still have a finite $0 \mathrm{~K}$ entropy clearly related to a dual dimer problem on a ladder (Fig. 2).

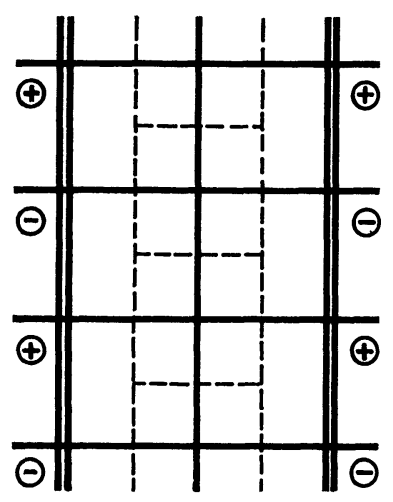

Fig. 2. - Frustration of the PUD model $\left(J^{\prime}<-J\right)$ : the dashed lines show the dual lattice available for dimers associated to unsatisfied bonds. The infinitely probable spin configuration for odd strings has been represented.

Now consider a vertical even string sandwiched between two antiferromagnetically ordered odd strings. If we assume the antiferromagnetic strings are in phase opposition, only two overall configurations are allowed for the intermediate even string. On the contrary when the odd strings are in phase there exists a large number of configurations, of the form $\alpha^{M}, M$ being the length of the string. This implies that in almost all $\left({ }^{2}\right)$ ground states, the antiferromagnetic strings are in phase and thus for these states there are only 2 configurations in so far as the spins in odd strings are considered (the degeneracy comes from the spins in even strings). Consequently almost all ground states fall into two classes, each class corresponding to one of the above defined configurations ; there is a one to one correspondence between the states in one class and the states in the other one

$\left.{ }^{2}\right)$ Almost all means all ground states except for a number which is an infinitely small fraction of the total number when the size of the system goes to infinity. by total spin reversal. Thus one can define an order parameter field

$$
\eta_{i}=\operatorname{Tr}_{\text {class } 1} S_{i}=-\operatorname{Tr}_{\text {class } 2} S_{i} \neq 0 .
$$

This order parameter is saturated on the odd strings : $\left|\eta_{i}\right|=1$. One can calculate $\eta_{i}$ on the even strings : fixing the spins on the neighbouring odd strings in phase (Fig. 2), one is left with a reduced Hamiltonian for each even string :

$$
\mathscr{H}_{\mathrm{red}}=-J \sum_{n} S_{n} S_{n+1}-2 J \sum_{n}(-1)^{n} S_{n}
$$

(ferromagnetic chain under staggered field). The calculation of the magnetization is straightforward by transfer matrix techniques :

$$
\left\langle S_{n}\right\rangle=(-1)^{n} \times \frac{1}{\sqrt{5}} .
$$

From this point of view the order parameter on the vertical even strings is not saturated, but the whole system is found to be antiferromagnetically ordered at $0 \mathrm{~K}$.

The same calculation yields the entropy per spin of the complete square lattice

$$
S=\frac{1}{2} \ln \alpha=\frac{1}{4} \ln \frac{3+\sqrt{5}}{2} \simeq 0.2406 .
$$

Because of the degeneracy of the ground state the question of the symmetry breaking and of the field conjugate to the order parameter requires some comments. As a matter of fact, at $T=0$ strictly speaking, the system chooses one of the allowed states, so that $\left|\left\langle S_{i}\right\rangle\right|=1$. But when $T=0^{+}$, a mixing occurs between the states in each class. The quantities calculated from $\mathcal{H}_{\text {red }}$ are actually the limiting values when $T \rightarrow 0$. To get some idea about the very low temperature behaviour of the system we have calculated some properties of Hamiltonian $\mathcal{H}_{\text {red }}$ when $T \neq 0$. In so doing, we expect qualitatively correct results which, when $J^{\prime} \gg J$, become quantitatively exact (in the latter case, spins in odd strings are frozen when $T$ is small enough). In particular we switched on a staggered field $h_{\sim}$, looked upon the staggered magnetization $m_{\sim}$ and the corresponding susceptibility $\chi_{\sim}$. Results are plotted in figure 3 . We see that the meaning of formula (1) is precisely

$$
\lim _{T \rightarrow 0} \lim _{h_{\sim} \rightarrow 0}\left\langle S_{n}\right\rangle=(-1)^{n} \frac{1}{\sqrt{5}} .
$$

We also see that the differential staggered susceptibility is infinite as $T \rightarrow 0$. This is clear from

$$
\chi_{\sim}=\frac{1}{T} \sum_{n}(-1)^{n}\left[\left\langle S_{0} S_{n}\right\rangle-\left\langle S_{0}\right\rangle\left\langle S_{n}\right\rangle\right] .
$$

Since the string is not saturated the term between brackets is finite and $\chi_{\sim} \rightarrow \infty$ when $T \rightarrow 0$. This argu- 


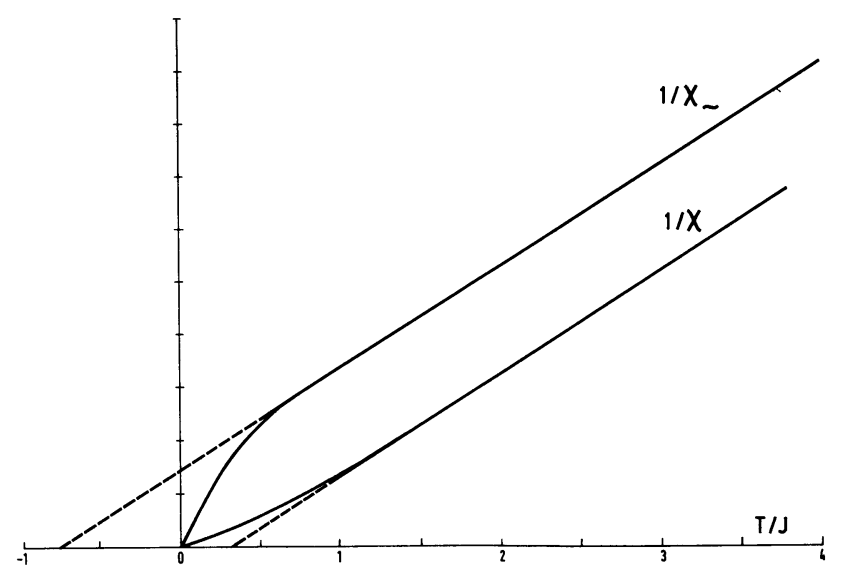

a)

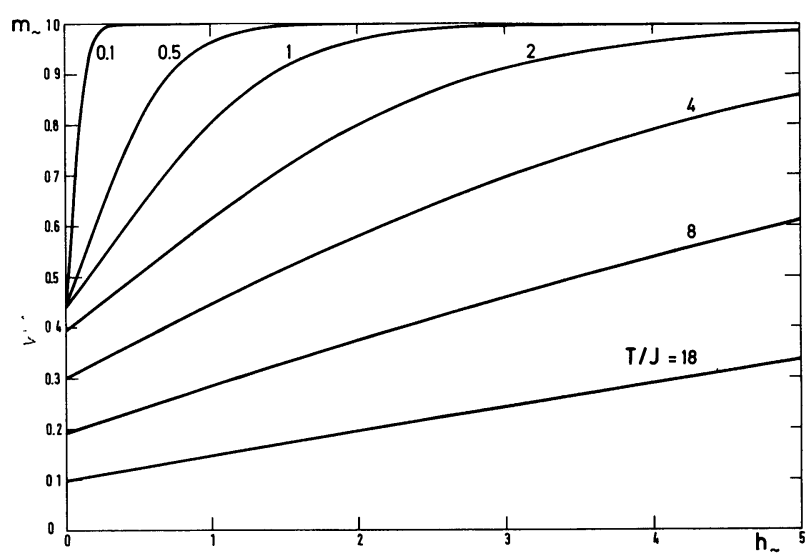

b)

Fig. 3. - a) Staggered and uniform susceptibilities versus temperature for Hamiltonian $\mathcal{H}_{\text {red }}$ (arbitrary units); b) Staggered magnetization versus staggered field for Hamiltonian $\mathcal{H}_{\text {red }}$ for different values of the temperature.

ment remains valid for almost any distribution of field : in particular we can show, using $\mathscr{H}_{\text {red }}$, that the uniform susceptibility $\chi$ diverges at $T=0$ (Fig. $3 a$ ).

2.2 Finite Temperature SOlution. - We first use the transfer matrix technique along the horizontal direction. We follow the method and notations of Lieb, Schultz, Mattis [3]; detailed calculations are given in Appendix I.
The transfer operator can be put into the form

$$
V_{\mathbf{H}}^{ \pm}=\exp \left(\text { const. }-\sum_{q} \varepsilon_{q} \xi_{q}^{\dagger} \xi_{q}\right)
$$

where the $\xi$ 's are fermion destruction operators and

$$
\begin{aligned}
& \varepsilon_{0}=4 K^{*}-2 K-2 K^{\prime} \\
& \varepsilon_{\pi}=4 K^{*}+2 K+2 K^{\prime} .
\end{aligned}
$$

For $q \neq\{0, \pi\}, \varepsilon_{q}$ is positive and given by $\cosh \varepsilon_{q}=\cosh 4 K^{*} \cosh 2\left(K+K^{\prime}\right)-\sinh 4 K^{*} \times$ $\times \sinh 2\left(K+K^{\prime}\right) \cos q-2 \sinh 2 K^{*}$ $\times \sinh 2 K^{\prime} \sin ^{2} q$.

One verifies that $\varepsilon_{q}$ can vanish only for $q=0$ and $q=\pi$. This occurs when

$$
\begin{array}{ll}
\sinh 2 K \sinh \left(K+K^{\prime}\right)=1 & \left(\varepsilon_{0}=0\right) \\
\sinh 2 K \sinh \left(K+K^{\prime}\right)=-1 & \left(\varepsilon_{\pi}=0\right) .
\end{array}
$$

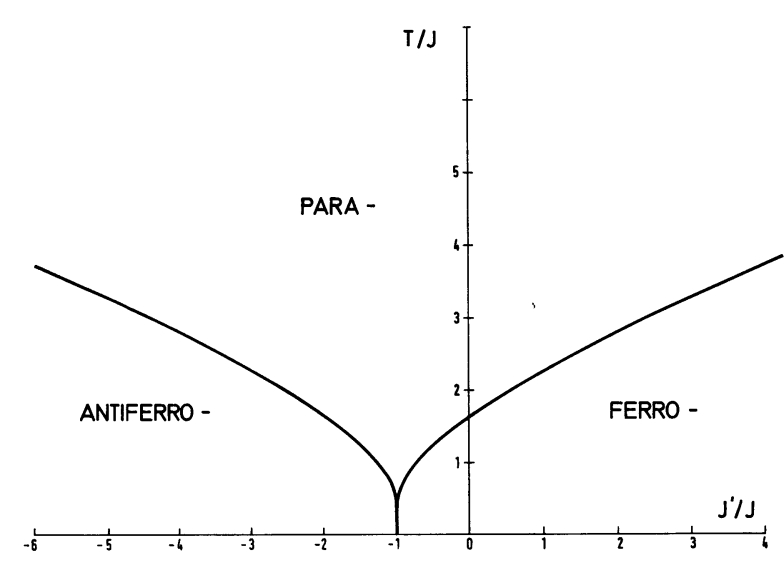

Fig. 4. - Phase diagram of the PUD model.

Thus we find 2 transition lines meeting at $T=0$, $J^{\prime}=-J$ (Fig. 4). Exactly as in the ordinary Ising model, below the transition the largest eigenvalue of the transfer operator is degenerate and separated by a gap from the other eigenvalues : this is interpreted as the onset of long range order according 'to the expressions of the horizontal correlation function in terms of eigenvalues $\Lambda_{\alpha}$ and eigenvectors $\psi_{\alpha}$ :

$$
G\left(x, y ; x^{\prime}, y\right)=\left\{\begin{array}{l}
\sum_{\alpha}\left|\left\langle\psi_{0}\left|\sigma_{0}^{z}\right| \psi_{\alpha}\right\rangle\right|^{2}\left(\Lambda_{\alpha} / \Lambda_{0}\right)^{\left|x-x^{\prime}\right| / 2} x, x^{\prime} \text { even } \\
\sum_{\alpha}\left\langle\psi_{0}\left|\tilde{\sigma}_{0}^{z}\right| \psi_{\alpha}\right\rangle\left\langle\psi_{\alpha}\left|\sigma_{0}^{z}\right| \psi_{0}\right\rangle\left(\Lambda_{\alpha} / \Lambda_{0}\right)^{\left(\left|x-x^{\prime}\right|+1\right) / 2} x \text { odd }, x^{\prime} \text { even } \\
\sum_{\alpha}\left|\left\langle\psi_{0}\left|\tilde{\sigma}_{0}^{z}\right| \psi_{\alpha}\right\rangle\right|^{2}\left(\Lambda_{\alpha} / \Lambda_{0}\right)^{\left|x-x^{\prime}\right| / 2} \quad x, x^{\prime} \text { odd }
\end{array}\right.
$$

where we have defined

$$
\tilde{\sigma}_{0}^{z}=V_{2}^{1 / 2}(K) V_{1}(K) \sigma_{0}^{z} V_{1}^{-1}(K) V_{2}^{-1 / 2}(K) .
$$

Spins on strings of the same parity are positively correlated along the horizontal direction, and the corresponding correlation length

$$
\xi_{\mathrm{H}}=\left(\ln \frac{\Lambda_{0}}{\Lambda_{1}}\right)^{-1}
$$

diverges at the transition point. 
As to the correlation between points belonging to strings of different parity, its sign is not clear from formula (2) but in view of the zero temperature results (Sec. 1) we shall regard it as positive at any temperature.

Now, to determine what type of L.R.O. is involved, we use the transfer matrix method along the vertical direction. The case of interest is indeed $J^{\prime}<0$, the system being obviously ferromagnetic for $J^{\prime}>0$. Here again the transfer operator appears as a product of $V_{q}^{\mathrm{v}}$ (see Appendix II). The complete diagonalization of $V_{q}^{\mathrm{v}}$ for all $q$ is far from easy $(16 \times 16$ matrix $)$; but we found that $V_{q}^{\mathrm{v}}$ is consistent with an expression of the type

$$
\begin{aligned}
V_{q}^{\mathrm{v}}=\exp [ & -E_{q}\left(u_{q}^{\dagger} u_{q}+u_{-q}^{\dagger} u_{-q}-1\right) \\
& \left.-E_{q}^{\prime}\left(v_{q}^{\dagger} v_{q}+v_{-q}^{\dagger} v_{-q}-1\right)\right](-1)^{\left(u_{q}^{\dagger} u_{q}+u_{-q}^{\dagger} u_{-q}\right)} \\
& \text { with } E_{q}, E_{q}^{\prime}>0 \quad \forall q \neq 0
\end{aligned}
$$

On the other hand we expect the singularity to come out of the term corresponding to $q=0$. The eigenvalues of the operator $V_{0}^{\mathrm{v}}$ are calculated in Appendix II and, identifying with expression (3),

$$
E_{0}=K^{*}+K^{\prime *}+\lambda, \quad E_{0}^{\prime}=K^{*}+K^{\prime \prime *}-\lambda
$$

with

$\sinh \lambda=\cosh 2 K \sinh \left(K^{\prime \prime *}-K^{*}\right)$.

For $-J \leqslant J^{\prime} \leqslant 0$ (resp. $\left.J^{\prime}<-J\right), E_{0}^{\prime}$ (resp. $E_{0}$ ) changes sign at the transition point previously defined; hence our conclusions :

i) for $J^{\prime}>-J$ the state vectors $\left|\psi_{0}^{+}\right\rangle$and $v_{0}^{\dagger}\left|\psi_{0}^{-}\right\rangle$(notations of Ref. [3]) are degenerate below $T_{\mathrm{c}}$ in the thermodynamic limit : the system is ferromagnetic ;

ii) for $J^{\prime}<-J$, the states $\left|\psi_{0}^{+}\right\rangle$and $u_{0}^{\dagger}\left|\psi_{0}^{-}\right\rangle$ are degenerate in the sense that they yield opposite eigenvalues. The vertical correlation develops a coherent part of the form $(-1)^{R}$ and finally we have a 4-sublattice antiferromagnet; hence the phase diagram of figure 4 .

The free energy per spin is

$$
F=-\frac{k T}{4}\left[\ln (2 \sinh 2 K)+\sum_{q}\left|\varepsilon_{q}\right|\right]
$$

For convenience it can be put into the form of a double integral :

$$
\begin{aligned}
-\beta F=\ln 2+ & \frac{1}{16 \pi^{2}} \int_{0}^{2 \pi} \int_{0}^{2 \pi} \mathrm{d} \theta \mathrm{d} \varphi \ln \left\{\frac { 1 } { 2 } \left[\cosh 2 K \cosh 2 K^{\prime}+\cosh ^{2} 2 K \cosh \left(K+K^{\prime}\right)-\right.\right. \\
& \left.\left.-\sinh ^{2} 2 K \cos \theta-2 \cosh 2 K \sinh 2\left(K+K^{\prime}\right) \cos \varphi+\sinh 2 K \sinh 2 K^{\prime} \cos 2 \varphi\right]\right\}
\end{aligned}
$$

This form could have been directly derived from a Pfaffian as well. The specific heat calculated from (4) has a logarithmic singularity at $T_{\mathrm{c}}$ as in the usual case, except for $J^{\prime}=-J$.

In summary the PUD model exhibits for $J+J^{\prime}<0$ ordinary antiferromagnetic order in so far as we consider its behaviour above and a little below the transition point. The unusual feature is the high ground state degeneracy leading to a non vanishing entropy and infinite susceptibilities at zero temperature. In particular the susceptibility of the staggered magnetization shows the very peculiar behaviour of figure 5 .

We may interpret our results by pointing out the existence of an infinite set $\mathcal{R}$ of rigid spins which we define as spins correlated in the same way in almost all ground states; in other words

$$
\mathbf{r} \in \mathcal{R}, \quad \mathbf{r}^{\prime} \in \mathcal{R} \Leftrightarrow\left\langle S_{\mathbf{r}} S_{\mathbf{r}^{\prime}}\right\rangle_{T=0}^{2}=1 .
$$

Here the set $\mathcal{R}$ is composed of all odd strings. When the system orders, the distribution of the order parameter is unambiguous and unique in the set $\mathcal{R}$ (after breaking of symmetry). The spins in $\mathcal{R}$ act like an internal field upon the remaining spins - which otherwise would be rather free - and induce order on them. Let us emphasize that the set $\mathcal{R}$ does not order by itself but requires a mediated interaction via

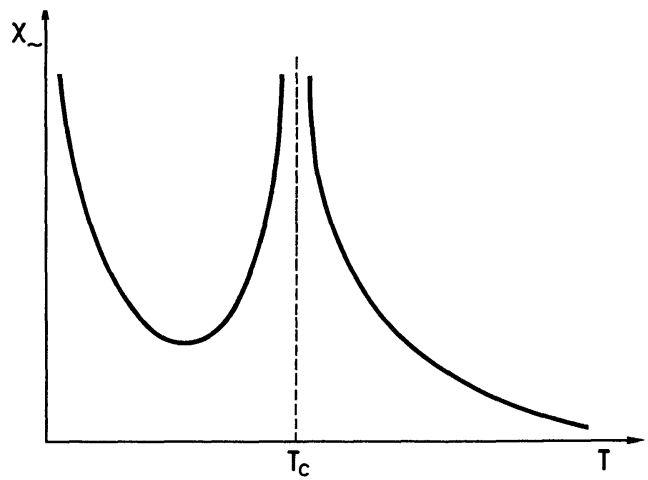

Fig. 5. - Shape of the staggered susceptibility of the PUD model $\left(J^{\prime}<-J\right)$ versus temperature.

its complementary $\bar{R}$. In particular $\mathcal{R}$ need not be a percolating set. At zero temperature this mediated interaction can be viewed as an entropy coupling. $\mathcal{R}$ determines the nature of the ordering at the critical point, whereas $\bar{R}$ is responsible for the anomalous low-temperature behaviour. We believe that this situation may be of some relevance to the spin glass problem, at least in the Ising case : a spin glass would be a magnet with a non periodic set $\mathcal{R}$.

It is interesting to mention that this model has been 
studied by Derrida et al. [6] using the approach of interface energy. In particular in this interpretation the existence of a transition is ascribed to a negative interface entropy at low $T$ (due to entropy coupling) rather than to the finite total entropy.
3. Solution of the zig-zag dominoes model. The partition function is calculated with the help of the Pfaffian technique. Some details of the involved algebra are given in Appendix III. The expression finally obtained is

$$
\begin{array}{r}
\ln Z=\ln 2+\frac{1}{16 \pi^{2}} \int_{0}^{2 \pi} \mathrm{d} \theta \int_{0}^{2 \pi} \mathrm{d} \varphi \ln \left[\cosh ^{2}\left(K+K^{\prime}\right) \cosh ^{2} 2 K-\sinh 2 K \sinh 2 K^{\prime}\left(\cos \theta \cos \varphi+\cos ^{2} \theta\right)-\right. \\
\left.-\sinh ^{2} 2 K\left(\cos \theta \cos \varphi+\cos ^{2} \varphi\right)\right]
\end{array}
$$

When $J^{\prime}=-J,\left(K^{\prime}=-K\right)$, the above expression reduces to

$$
\ln Z=\ln 2+\frac{1}{4 \pi^{2}} \int_{0}^{\pi} \mathrm{d} \theta \int_{0}^{\pi} \mathrm{d} \varphi \ln \left[1+\sinh ^{2} 2 K \times\left(\cos ^{2} \theta+\sin ^{2} \varphi\right)\right] .
$$

3.1 $J^{\prime}>-J .-$ In this case, like in the PUD model, the ground state is non degenerate, and ferromagnetic. Inspection of expression (5) shows that the system exhibits a transition point defined by

$$
2 \tanh \left(K+K^{\prime}\right) \tanh 2 K=1 .
$$

The system behaves like the standard ferromagnetic Ising model : the specific heat displays a logarithmic singularity, and all the critical exponents are expected to be standard too. The corresponding phase diagram in $\left(J^{\prime} / J, T / J\right)$ coordinates is presented in figure 6.

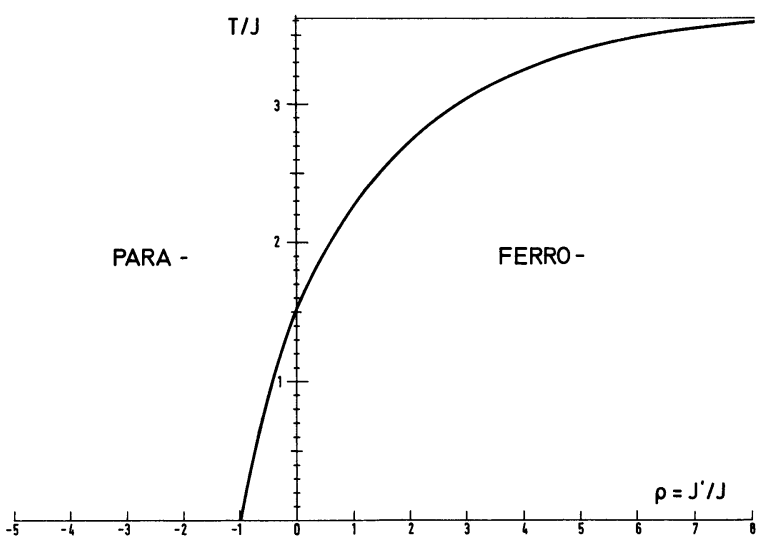

Fig. 6. - Phase diagram of the ZZD model.

The horizontal asymptote observed for $J^{\prime} / J \rightarrow \infty$ describes the equivalence of our model, in this limit, with a ferromagnetic triangular model whose interactions are all equal to $J:$ the spins of each pair connected with a $J^{\prime}$ bond are parallel and may be replaced by a fictitious spin and the corresponding bond deleted, giving a triangular lattice with $N / 2$ spins.

$3.2 J^{\prime}=-J$. - Because of the Mattis transform relating the PUD and ZZD models, both of them have the same residual entropy that can be calculated from (6) :

$$
\begin{aligned}
S & =\frac{1}{4 \pi^{2}} \int_{0}^{\pi} \mathrm{d} \theta \int_{0}^{\pi} \mathrm{d} \varphi \ln \left[4\left(\cos ^{2} \theta+\sin ^{2} \varphi\right)\right] \\
& =\frac{G}{\pi}=0.2916
\end{aligned}
$$

( $G$ is Catalan's constant ; we recover the entropy of a dimer assembly), and none of them orders. The specific heat is a smoothly varying function of $T$, and behaves at low temperature like $\frac{1}{T^{3}} \exp -4 J / T$.

3.3 $J^{\prime}<-J$. - Like in the PUD model, in any ground state the pairs of spins coupled by $J^{\prime}$ are antiferromagnetically arranged so that the frustration effect is left on the $J$ bonds : it amounts to a dimer problem on an hexagonal lattice (Fig. 7). Here again

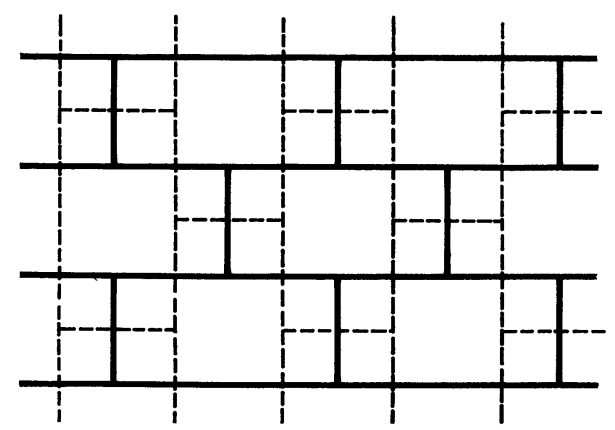

Fig. 7. - Frustration of the ZZD model $\left(J^{\prime}<-J\right)$; the dashed lines show the (hexagonal) dual lattice available for dimers associated to unsatisfied bonds.

we have a residual entropy at $T=0$, which turns out to be the same as that for the antiferromagnetic triangular lattice with $N / 2$ sites, for, in so far as we look only for ground states, the above mentioned pair can be replaced by a fictitious Ising spin (say +1 for $(+-)$ and -1 for $(-+)$ ), and it is readily seen that the effective interaction between fictitious 
spins is $-J$ distributed on a triangular lattice. Indeed from (5) one finds

$$
S=\frac{1}{4 \pi^{2}} \int_{0}^{\pi} \mathrm{d} \theta \int_{0}^{\pi} \mathrm{d} \varphi \ln \left[1+4 \cos \theta \cos \varphi+4 \cos ^{2} \theta\right]
$$

which is half the value obtained by Wannier [4].

Unlike the PUD model, the partition function for $J^{\prime} \leqslant-J$ has no singularity and we conclude that the model is disordered at any finite temperature. The possibility of a L.R.O. at $T=0$ is still an open question.

Before closing this section concerning the ZZD model, the topological role of the $J^{\prime} / J=\rho$ ratio should be emphasized. When $\rho$ takes respectively the values $-\infty,-1,0,1,+\infty$, we recover successively the triangular antiferromagnetic, purely frustrated, hexagonal fersomagnetic, square ferromagnetic and triangular ferromagnetic Ising models, in a somewhat continuous manner as a function of $\rho$.

4. The special case $J^{\prime}=-J$. Dilute frustrated plaquettes. - As already mentioned for $J^{\prime}=-J$ the zig-zag dominoes model and the piled up dominoes model are equivalent. This is precisely the case for which frustration was originally introduced $[1,5,7]$. We showed there is no transition temperature but the nature of the correlation is an interesting question.

Consider the horizontal transfer matrix of the PUD model for $K+K^{\prime}=0, T=0$. We have

$$
\cosh \varepsilon_{q}=1+2 \sin ^{2} q
$$

and thus the spectrum of $V_{\mathrm{H}}$ is a continuum just above the 3-fold degenerate ground state (no gap). This is -reminiscent of the situation at the finite temperature transition point of an ordinary, say, Ising model, in which the correlation length is infinite and the correlation decreases slowly following a power law. So it is likely that in the PUD model the horizontal correlation behaves like

$$
\left.G_{\mathrm{H}}(R)\right|_{\substack{T=0 \\ J^{\prime}=-J}} \sim \frac{1}{R^{d-2+\eta}}
$$

at $T=0$.

Unfortunately the $\eta$ exponent does not come out simply of our formalism : this point will be the matter of a later study together with the interesting question of the zero temperature correlation of the ZZD model in the disordered region.

We now notice that the vertical correlation $G_{\mathrm{V}}$ can be expressed from $G_{\mathrm{H}}$ with the help of the gauge transform $\varepsilon$ defined on figure 8 and which amounts to a $\pi / 2$ rotation of the lattice :

$$
G(\mathbf{r}, \mathbf{r}+R \mathbf{b})=\varepsilon_{\mathbf{r}} \varepsilon_{\mathbf{r}+R \mathbf{b}} G(\mathbf{r}, \mathbf{r}+R \mathbf{a})
$$

and, apart from a sign, $G_{\mathrm{V}}$ behaves like $G_{\mathrm{H}}$ :

$$
\left|G_{\mathrm{v}}(R)\right| \sim \frac{1}{R^{d-2+\eta}}
$$

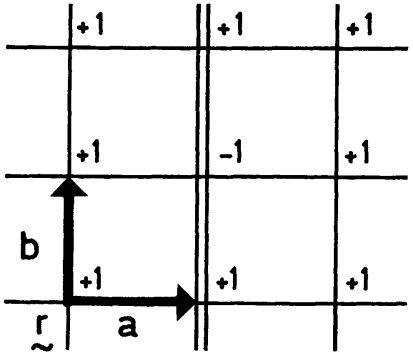

Fig. 8. - Gauge transform $\varepsilon$ changing a vertical PUD into a horizontal one.

In conclusion, the introduction of a different interaction $J^{\prime}$ helps us guess the properties of the odd model : at $T=0$ it is quasi-ordered, and, more precisely, involves a mixing of ferro- and antiferromagnetic quasi-order (tricritical point).

The above conclusion about the totally frustrated model can be partially extended to a model involving periodically dilute frustrated plaquettes as shown in figure 9 : the frustrated plaquettes form a square superstructure of period $n$ embedded in normal plaquettes. The interactions are assumed to be $J_{i j}= \pm 1$

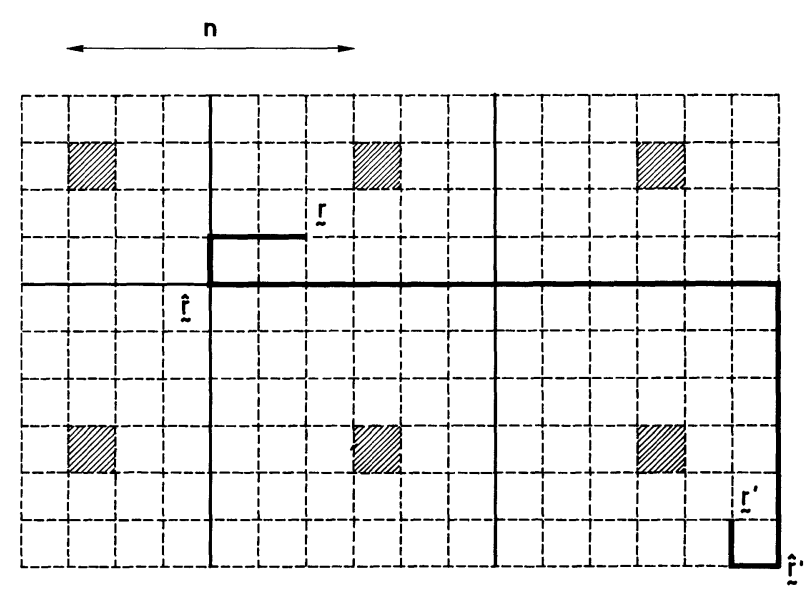

Fig. 9. - Square superstructure of dilute frustrated plaquettes. The frustrated plaquettes are shaded, the superlattice is represented in full lines, the path used for the correlation between $\mathbf{r}$ and $\mathbf{r}^{\prime}$ in heavy lines.

Let us define a superlattice dividing the original lattice into cells so that each cell contains one and only one frustrated plaquette. The zero degree entropy per spin of this model is clearly equal to the entropy of a Villain model defined on the superlattice, namely $\frac{1}{n^{2}} \frac{G}{\pi}$. Moreover a similar connection holds for the correlation function. Given a site $\mathbf{r}$ of the lattice we define $\hat{\mathbf{r}}$ as the nearest site of the superlattice : rigorously it means there is a path irom $\mathbf{r}$ to $\hat{\mathbf{r}}$ which is not cut by any superdimer connecting the frustrated plaquettes in any ground state. Consider a path between two sites $\mathbf{r}$ and $\mathbf{r}^{\prime}$ passing by $\hat{\mathbf{r}}$ and $\hat{\mathbf{r}}^{\prime}$ such that between 
$\hat{\mathbf{r}}$ and $\hat{\mathbf{r}}^{\prime}$ this path is drawn on the superlattice. We have for the correlation at $T=0$

$$
G\left(\mathbf{r}, \mathbf{r}^{\prime}\right)=\left(\prod_{\text {path }} J_{i j}\right)\left\langle(-1)^{N\left(\mathbf{r}, \mathbf{r}^{\prime}\right)}\right\rangle
$$

$N\left(\mathbf{r}, \mathbf{r}^{\prime}\right)$ is the number of times the path is cut by superdimers. If the intracell end parts of the path, from $\mathbf{r}$ to $\hat{\mathbf{r}}$ and from $\hat{\mathbf{r}}^{\prime}$ to $\mathbf{r}^{\prime}$, are chosen as explained above (i.e. not cut by superdimers),

$$
G\left(\mathbf{r}, \mathbf{r}^{\prime}\right)=\left(\prod_{\text {path }} J_{i j}\right)\left\langle(-1)^{N\left(\hat{r}, \hat{\mathbf{r}}^{\prime}\right)}\right\rangle .
$$

The quantity between brackets gives the correlation between $\hat{\mathbf{r}}, \hat{\mathbf{r}}^{\prime}$ in the totally frustrated model defined on the superlattice (interactions \pm 1 ) namely $G_{0}\left(\hat{\mathbf{r}}, \hat{\mathbf{r}}^{\prime}\right)$; so

$$
\left|G\left(\mathbf{r}, \mathbf{r}^{\prime}\right)\right|=\left|G_{0}\left(\hat{\mathbf{r}}, \hat{\mathbf{r}}^{\prime}\right)\right| .
$$

For large distances, the relation is a simple change of scale

$$
|G(R)| \sim\left|G_{0}(R / n)\right| .
$$

We may infer that the model is quasi-ordered at $T=0$ (same exponent $\eta$ ) and thus exhibits no finite transition temperature.

We believe this is due to the absence of rigid spins in this model. This view is supported by another model we can call the chess-board model and which is defined in figure 10 . We have calculated exactly the partition function and found no transition, as expected because of the absence of rigid spins (some

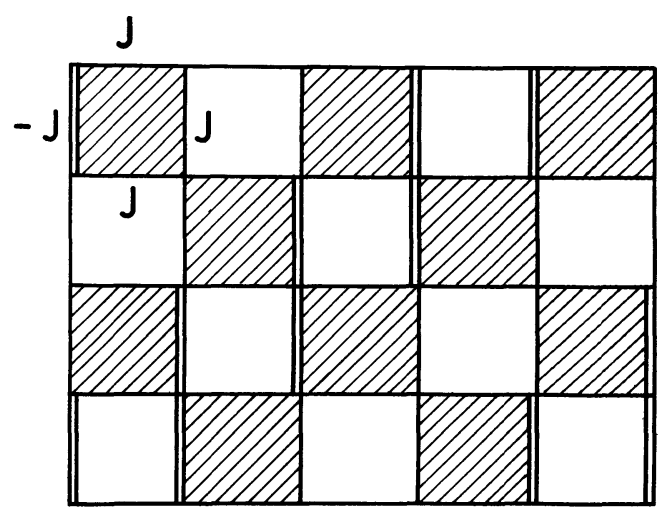

Fig. 10. - The chess-board model.

indications and comments can be found in Appendix IV).

In conclusion let us emphasize that our hypothesis about the necessity of rigid spins for observing a phase transition implies the non existence of a spin glass state in periodic frustrated systems since in this case the set of rigid spins $\mathcal{R}$ is necessarily periodic, which in turn induces periodic order at zero temperature. This conclusion is, a priori, not restricted to two-dimensional systems.

Acknowledgments. - The authors wish to acknowledge many fruitful discussions with Drs. Aubry, Derrida, Maillard, Pomeau, Sarma, Vannimenus. Litteral expansion of $16 \times 16$ determinants through computer techniques was achieved with the help of Dr. Raynal who provided the suitable mathematical method [9], and to whom we are especially indebted.

\section{Appendix I}

HORIZONTAL TRANSFER MATRIX (PUD MODEL). - The transfer matrix formalism applies in a straightforward manner to the PUD model when the transfer direction is chosen to be horizontal (Fig. 1). The reader is referred to the paper by Lieb, Schultz and Mattis. The transfer operator is then

$$
V_{\mathrm{H}}=V_{2}^{1 / 2}(K) V_{1}(K) V_{2}\left(K^{\prime}\right) V_{1}(K) V_{2}^{1 / 2}(K)
$$

with

$$
\begin{gathered}
K=\beta J \quad K^{\prime}=\beta J^{\prime} \\
V_{1}(K)=\prod_{i}\left(\mathrm{e}^{K}+\mathrm{e}^{-K} \sigma_{i}^{x}\right)=(2 \sinh 2 K)^{M / 2} \exp K^{*} \sum_{i} \sigma_{i}^{x} \\
V_{2}(K)=\exp K \sum_{i} \sigma_{i}^{z} \sigma_{i+1}^{z} .
\end{gathered}
$$

The suscript $i$ runs on the $M$ spins of a vertical chain.

After Jordan-Wigner, then Fourier transforms, we get

$$
V_{\mathrm{H}}^{ \pm}=(2 \sinh 2 K)^{M / 2} \prod_{0 \leqslant q \leqslant \pi} V_{q}^{\mathrm{H}}
$$

where

$$
\begin{aligned}
V_{q}^{\mathrm{H}} & =V_{2 q}^{1 / 2}(K) V_{1 q}(K) V_{2 q}\left(K^{\prime}\right) V_{1 q}(K) V_{2 q}^{1 / 2}(K) \\
V_{1 q} & =\exp -2 K^{*}\left(\eta_{q}^{\dagger} \eta_{q}+\eta_{-q}^{\dagger} \eta_{-q}-1\right) \\
V_{2 q} & =\exp 2 K\left[(\cos q)\left(\eta_{q}^{\dagger} \eta_{q}+\eta_{-q}^{\dagger} \eta_{-q}\right)+(\sin q)\left(\eta_{q} \eta_{-q}+\eta_{-q}^{\dagger} \eta_{q}^{\dagger}\right)\right]
\end{aligned}
$$


We first observe that

$$
\begin{aligned}
& V_{0}^{\mathbf{H}}=\exp \left[-\left(4 K^{*}-2 K-2 K^{\prime}\right) \eta_{0}^{\dagger} \eta_{0}+K^{*}\right] \\
& V_{\pi}^{\mathbf{H}}=\exp \left[-\left(4 K^{*}+2 K+2 K^{\prime}\right) \eta_{\pi}^{\dagger} \eta_{\pi}+K^{*}\right] .
\end{aligned}
$$

We now turn to the case $q \neq\{0, \pi\}$. As in the usual case $\phi_{q}$ and $\phi_{-q}$ are trivial eigenvectors of $V_{q}^{\mathbf{H}}$

$$
V_{q}^{\mathrm{H}} \phi_{q}=\left[\exp 2\left(K+K^{\prime}\right) \cos q\right] \phi_{q} .
$$

Thus we need the matrix of $V_{q}$ in the subspace spanned by $\phi_{0}$ and $\phi_{-q q}$. In this subspace we have

$$
\begin{aligned}
\text { mat } V_{1 q}(K) & =\left[\begin{array}{cc}
\mathrm{e}^{-2 K^{*}} & 0 \\
0 & \mathrm{e}^{2 K^{*}}
\end{array}\right] \\
\mathrm{e}^{-2 K \cos q} \times \operatorname{mat} V_{2 q}(K) & =\left[\begin{array}{cc}
\cosh 2 K+\sinh 2 K \cos q & \sinh 2 K \sin q \\
\sinh 2 K \sin q & \cosh 2 K-\sinh 2 K \cos q
\end{array}\right] .
\end{aligned}
$$

All these matrices are of determinant unity. Thus we can write

$$
V_{q}^{\mathbf{H}}=\exp \left[2\left(K+K^{\prime}\right) \cos q\right] \exp -\varepsilon_{q}\left(\xi_{q}^{\dagger} \xi_{q}+\xi_{-q}^{\dagger} \xi_{-q}-1\right)
$$

where $\varepsilon_{q}$ is given by

$$
2 \cosh \varepsilon_{q}=\exp \left[-2\left(K+K^{\prime}\right) \cos q\right] \operatorname{Tr} \text { mat } V_{q}^{\mathbf{H}}
$$

and is always positive. The trace of mat $V_{q}^{\mathrm{H}}$ is obtained by elementary algebra :

$\cosh \varepsilon_{q}=\cosh 4 K^{*} \cosh 2\left(K+K^{\prime}\right)-\sinh 4 K^{*} \sinh 2\left(K+K^{\prime}\right) \cos q-2 \sinh 2 K^{*} \sinh 2 K^{\prime} \sin ^{2} q$.

\section{Appendix II}

VeRTICAL TRANSFER MATRIX (PUD MODEL). - For $J^{\prime}<0$ we set $K^{\prime \prime}=-\beta J^{\prime}>0$. The transfer matrix is written

and

$$
V_{\mathrm{v}}=V_{2}^{1 / 2} W_{1} V_{2}^{1 / 2} \quad \text { with } \quad V_{2}=\exp K \sum_{i} \sigma_{i}^{z} \sigma_{i+1}^{z}
$$

$$
\begin{aligned}
W_{1} & =\prod_{n}\left(\mathrm{e}^{K}+\mathrm{e}^{-K} \sigma_{2 n}^{x}\right)\left(\mathrm{e}^{-K^{\prime \prime}}+\mathrm{e}^{K^{\prime \prime}} \sigma_{2 n+1}^{x}\right) \\
& =\left(4 \sinh 2 K \sinh 2 K^{\prime \prime}\right)^{N / 2} \exp \left(K^{*} \sum_{n} \sigma_{2 n}^{x}+K^{\prime \prime} * \sum_{n} \sigma_{2 n+1}^{x}\right) \prod_{n} \sigma_{2 n+1}^{x} .
\end{aligned}
$$

There are two spins per unit cell, so we must introduce 2 kinds of Fermi operators $a_{k}$ and $b_{k}$, which allows us to write

$$
\begin{gathered}
V_{\mathrm{v}}^{ \pm}=\left(4 \sinh 2 K \sinh 2 K^{\prime \prime}\right)^{N / 2} \prod_{0 \leqslant k \leqslant \pi / 2} W_{2 k}^{1 / 2} W_{1 k} W_{2 k}^{1 / 2} \\
W_{1 k}=\exp \left[-2 K^{*}\left(a_{k}^{\dagger} a_{k}+a_{-k}^{\dagger} a_{-k}-1\right)-2 K^{\prime \prime *}\left(b_{k}^{\dagger} b_{k}+b_{-k}^{\dagger} b_{-k}-1\right)\right] \times(-1)^{\left(b_{k}^{\dagger} b_{k}+b_{-k}^{\dagger} b-k\right)} \\
W_{2 k}=\exp 2 K\left[(\cos k)\left(a_{k}^{\dagger} b_{k}+a_{-k}^{\dagger} b_{-k}\right)-(i \sin k)\left(b_{k} a_{-k}-b_{-k} a_{k}\right)+\text { h.c. }\right] .
\end{gathered}
$$

We look at the term at $q=0$, namely $V_{0}^{\mathrm{v}}$. This operator involves only four state vectors, two of which being trivial eigenvectors

$$
V_{0}^{\mathrm{v}}|0\rangle=\mathrm{e}^{K^{*}+K^{\prime *}}|0\rangle, \quad V_{0}^{\mathrm{v}} a_{0}^{\dagger} b_{0}^{\dagger}|0\rangle=-\mathrm{e}^{-K^{*}-K^{\prime *}} a_{0}^{\dagger} b_{0}^{\dagger}|0\rangle
$$

where $|0\rangle$ is the vacuum state of $a_{0}$ and $b_{0}$. Between the states $a_{0}^{\dagger}|0\rangle$ and $b_{0}^{\dagger}|0\rangle$ the matrix of $V_{0}^{\mathrm{v}}$ is

$$
\left[\begin{array}{cc}
\cosh ^{2} K \mathrm{e}^{K^{\prime *}-K^{*}}-\sinh ^{2} K \mathrm{e}^{K^{*}-K^{\prime *}} & 2 \sinh K \cosh K \sinh \left(K^{\prime \prime *}-K^{*}\right) \\
2 \sinh K \cosh K \sinh \left(K^{\prime *}-K^{*}\right) & -\cosh ^{2} K \mathrm{e}^{K^{*}-K^{\prime *}}+\sinh ^{2} K \mathrm{e}^{K^{\prime *}-K^{*}}
\end{array}\right] .
$$

Its determinant is -1 , its eigenvalues $\mathrm{e}^{\lambda}$ and $-\mathrm{e}^{-\lambda}, \lambda$ being given by

$$
\sinh \lambda=\cosh 2 K \cdot \sinh \left(K^{\prime \prime *}-K^{*}\right) \text {. }
$$




\section{Appendix III}

PARTITION FUnCTION OF THE ZZD MODEL. - The conventional Pfaffian method is used [8]. The lattice ( $n$ rows and $m$ columns, $m n=N$ ) is helically wound on a torus. The set of the interactions may be described via a unit cell (represented in figure 11a) translated $N / 2$ times along the helix. The ZZD configuration is obtained by imposing $m$ odd, $n$ even (note that $m$ even, $n$ indifferent leads to the PUD configuration). The partition function $Z^{N}$ for the $N$ spins of the ZZD model is then given by

$$
Z^{2 N}=\left(2 \cosh ^{3 / 2} K \cosh ^{1 / 2} K^{\prime}\right)^{2 N} \prod_{\omega} D(\omega)
$$

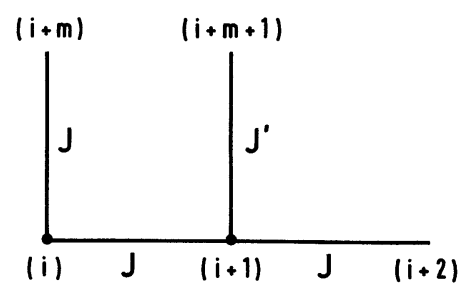

Fig. 11a. - The elementary cell of interactions in the ZZD model, as introduced in the Pfaffian technique.

where $D(\omega)$ is an $8 \times 8$ determinant :

$$
D(\omega)=\left|\begin{array}{rrrrrrrc}
0 & 1 & -1 & 1 & 0 & 0 & -x & 0 \\
-1 & 0 & 1 & -1 & 0 & 0 & 0 & -\omega^{(m-1) / 2} x \\
1 & -1 & 0 & 1 & -\omega^{-1} x & 0 & 0 & 0 \\
-1 & 1 & -1 & 0 & 0 & \omega^{-(m+1) / 2} y & 0 & 0 \\
0 & 0 & -\omega x & 0 & 0 & 1 & -1 & 1 \\
0 & 0 & 0 & -\omega^{(m+1) / 2} y & -1 & 0 & 1 & -1 \\
x & 0 & 0 & 0 & 1 & -1 & 0 & 1 \\
0 & \omega^{-(m-1) / 2} x & 0 & 0 & -1 & 1 & -1 & 0
\end{array}\right|
$$

where $x=\tanh K, y=\tanh K^{\prime}$, and $\omega$ runs on all the $(N / 2)$ th roots of -1 . After a $4 \times 4$ block triangularization, one easily finds

$$
D(\omega)=\operatorname{det}\left|\begin{array}{cccc}
0 & 1-\omega^{-(m-1) / 2} x y & -\left(1+\omega x^{2}\right) & 1-\omega^{(m+1) / 2} x^{2} \\
-\left(1-\omega^{(m-1) / 2} x y\right) & 0 & 1-\omega^{(m+1) / 2} x y & -\left(1+\omega^{m} x y\right) \\
1+\omega^{-1} x^{2} & -\left(1-\omega^{-(m+1) / 2} x y\right) & 0 & 1-\omega^{(m-1) / 2} x^{2} \\
-\left(1-\omega^{-(m+1) / 2} x^{2}\right) & 1+\omega^{-m} x y & -\left(1-\omega^{-(m-1) / 2} x^{2}\right) & 0
\end{array}\right|
$$

In the limit of large values of $m$ and $n$, we perform the usual substitution

with

$$
\begin{aligned}
\omega & =\exp (2 i \varphi), & \omega^{m} & =\exp (2 i \theta) \\
\varphi & =\left(\frac{j-1}{m}\right) \pi, & j & =1,2, \ldots, m \\
\theta & =\left(\frac{2 k-1}{n}\right) \pi, & k & =1,2, \ldots, n / 2
\end{aligned}
$$

and

but attention must be paid to the fact that $\omega^{m / 2}=\mathrm{e}^{i \theta}$ or $\mathrm{e}^{i(\theta+\pi)}$ according as index $j$ is odd or even (these two determinations will be equally represented in the continuum limit).

This provides the expansion of $D(\omega)$ as defined in (A3.3) in the form $D(\omega)=D(\theta, \varphi)(\operatorname{resp} . D(\theta+\pi, \varphi))$, with

$$
\begin{aligned}
D(\theta, \varphi)=\left(1+x^{2}\right)^{2}(1+x y)^{2}-4 x y\left(1-x^{2}\right)^{2} & \left(\cos \theta \cos \varphi+\cos ^{2} \theta\right)- \\
& -4 x^{2}\left(1-x^{2}\right)\left(1-y^{2}\right)\left(\cos \theta \cos \varphi+\cos ^{2} \varphi\right) .
\end{aligned}
$$

Insertion of this expression into (A3.1) yields

$$
\ln Z=\ln 2+\ln \left(\cosh ^{3 / 2} K \cosh ^{1 / 2} K^{\prime}\right)+\frac{1}{16 \pi^{2}} \int_{0}^{2 \pi} \int_{0}^{2 \pi} D(\theta, \varphi) \mathrm{d} \theta \mathrm{d} \varphi .
$$


Insertion of $\ln \left(\cosh ^{3 / 2} K \cosh ^{1 / 2} K^{\prime}\right)$ into the integral, followed by elementary manipulations on the hyperbolic terms leads to the required expression as given in (5).

\section{Appendix IV}

PARTITION FUnCtion OF THE " CHESS-BOARD " MODEL. - Lines analogous to those described in (A.3) are followed. The unit cell of interactions (Fig. $11 b$ ) is translated $N / 4$ times along the helix ; $m$ and $n$ are chosen so that $n / 4$ and $(m+1) / 4$ should be integers.

The resulting determinantal algebra is cumbersome and will not be reproduced here (the elementary determinants appearing in the antisymmetrical Pfaffian are now $16 \times 16$; the general case $J^{\prime} \neq J$ has been dealt with).

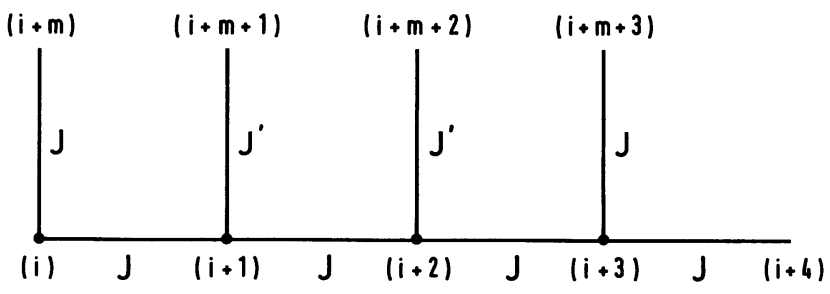

Fig. 11b. - The elementary cell of interactions in the chess-board model, as introduced in the Pfaffian technique.

In the particular case $J^{\prime}=-J$ one finds

$\ln Z=\ln 2+\frac{1}{32 \pi^{2}} \int_{0}^{2 \pi} \int_{0}^{2 \pi} \ln \left\{\frac{\left(x^{4}+1\right)^{2}+4 x^{6}+8 x^{4}+4 x^{2}}{\left(1-x^{2}\right)^{4}}+\frac{4 x^{4}}{\left(1-x^{2}\right)^{4}} \times\right.$
$\left.\times\left[-1+(\sin \theta+\sin \varphi)^{2}\right]\right\} \mathrm{d} \theta \mathrm{d} \varphi$

where $x=\tanh K$.

At $0 \mathrm{~K}$, a residual entropy per spin

$$
S(0)=\frac{1}{32 \pi^{2}} \int_{0}^{2 \pi} \int_{0}^{2 \pi} \ln \left[16+4(\sin \theta+\sin \varphi)^{2}\right] \mathrm{d} \theta \mathrm{d} \varphi
$$

is found. Obviously, $1 / 2 \ln 2<S(0)<5 / 8 \ln 2$ : the residual entropy in this case is larger than in the ZZD problem.

Inspection of (A4.1) shows that the argument of the logarithm in the integral is strictly positive and continuous with respect to each of the $T, \theta, \varphi$ variables except at $T=0$. Therefore no transition at finite temperature occurs. The situation at $T=0$ is ambiguous, like in the ZZD model.

\section{References}

[1] Toulouse, G., Commun. Phys. 2 (1977) 115.

[2] Villain, J., J. Phys. C : Solid State Phys. 10 (1977) 1717.

[3] Lieb, E. H., Schultz, T. D., Mattis, D. C., Rev. Mod. Phys. 36 (1964) 856.

[4] Wannier, G. H., Phys. Rev. 78 (1950) 341.

[5] Vannimenus, J., Toulouse, G., J. Phys. C; Solid State Phys. 10 (1977) L-537.
[6] Derrida, B., Maillard, J. M., Vannimenus, J., Kirkpatrick, S., J. Physique Lett. 39 (1978) L-465.

[7] Kirkpatrick, S., Phys. Rev. B 16 (1977) 4630.

[8] Green, H. S., Hurst, C. A., Order disorder phenomena (J. Wiley \& Sons) 1964, Chapt. 3.

[9] Raynal, J., J. Physique 31 (1970) 3. 